\title{
Avaliação do funcionamento de barraginhas em solos de textura média e arenosa
}

\author{
Victor Rodrigues Aragão '; Adrielle Ferreira Brito '; Ana Clara Soares de Souza ${ }^{2}$; Emílio Rodrigues \\ Versiani Júnior ${ }^{2}$
}

凹aragaorvictor@gmail.com

1. Instituto Educacional Santo Agostinho (IESA) 2. Universidade Federal de Minas Gerais (UFMG).

\section{Histórico do Artigo:}

Recebido em: 04 de março de 2019

Resumo: 0 presente estudo buscou avaliar o funcionamento de diferentes barraginhas na comunidade de Abóboras, no estado brasileiro de Minas Gerais, em solos de texturas média e arenosa. Como metodologia adotouse critérios a serem avaliados: condições atuais de dimensão, granulometria de solos para classificação quanto a texturas e determinação dos cálculos de velocidade de infiltração básica. Os resultados apontaram que as oito barraginhas avaliadas no estudo estão com seus funcionamentos comprometidos, sendo que as identificadas como número 3 e 8 apresentam taxas mais distantes da ideal (30 mm.h-l) 11,33 mm.h-1 para a barraginha 3 e 11,99 mm.h-1 para a 8. 0s dados obtidos referentes a texturas de solos e dimensões refletiram forte convergência às taxas de infiltração, levando à conclusão de que mais de $25 \%$ dos sistemas avaliados apresentam textura média granulométrica, e o acúmulo dos sedimentos pela falta de monitoramento se fez evidente, com redução da taxa média de profundidade de 1,5 m para 0,32 m em 11 anos. Foi possível concluir que os solos das barraginhas apresentam altas capacidades de infiltração, contudo, suas estruturas estão operando com sobrecarga, necessitando, portanto, de manutenção que deveria ser realizada com uma periodicidade de cinco anos.

Palavras-chave: Dimensão, granulometria de solos, velocidade de infiltração básica, sedimentos.

\section{Evaluation of the operation of infiltration dams in soils of medium and sandy texture}

\begin{abstract}
The present study sought to evaluate the functioning of different infiltration dams in the community of Abóboras, in the Brazilian state of Minas Gerais, in soils of medium and sandy texture. The methodology established considered the following criteria to be evaluated: current conditions of size, soil grading for classification of textures and determination of basic infiltration rate calculations. The results indicated that the eight dams evaluated in the study are compromised, with those identified as number 3 and 8 having rates farther from the ideal (30 mm.h-l) - $11.33 \mathrm{~mm} . \mathrm{h}-1$ for dam 3 and $11.99 \mathrm{~mm} . \mathrm{h}-1$ for dam 8 . Data obtained regarding soil textures and dimensions reflected a strong convergence to the infiltration rates, leading to the conclusion that more than $25 \%$ of the evaluated systems exhibited medium soil grading, and sediment accumulation due to lack of monitoring was evident, with a reduction in the mean depth from $1.5 \mathrm{~m}$ to $0.32 \mathrm{~m}$ in 11 years. It was possible to conclude that the soils of the systems have high infiltration capacities, however, their structures are operating with overload, requiring maintenance, which should be performed with a periodicity of 5 years.
\end{abstract}

Keywords: Dimension, soil grading, basic infiltration rate, sediment. 


\section{Evaluación de la operación de presas de infiltración en los suelos de textura media y arenosa}

Resumen: El presente estudio buscó evaluar el funcionamiento de diferentes represas de infiltración en la comunidad de Abóboras, en el estado brasileño de Minas Gerais, en suelos de textura media y arenosa. Estableció como metodología criterios a ser evaluados: condiciones actuales de dimensión, granulometría de suelos para clasificación en cuanto a texturas y determinación de los cálculos de velocidad de infiltración básica. Los resultados apuntaron que las ocho represas evaluadas en el estudio están con sus funcionamientos comprometidos, siendo que las identificadas como número 3 y 8 tienen tasas más distantes de la ideal (30 mm.h1) - 11,33 mm.h-1 para la represa 3 y 11,99 mm.h-1 para la 8 . Los datos obtenidos en función de texturas de suelos y dimensiones reflejaron fuerte convergencia a las tasas de infiltración, llevando a la conclusión de que más del 25\% de los sistemas evaluados presentaban textura granulométrica media, y la acumulación de sedimentos por la falta de monitoreo se hizo evidente, con reducción de la tasa media de profundidad de 1,5 m para $0,32 \mathrm{~m}$ en 11 años. Es posible concluir que los suelos de los sistemas presentan altas capacidades de infiltración, sin embargo, sus estructuras están operando con carga excesiva, necesitando, por lo tanto, de mantenimiento, que debería realizarse con una periodicidad de 5 años.

Palabras clave: Dimensión, granulometría de suelos, velocidad de infiltración básica, sedimentos.

\section{INTRODUÇ̃̃o}

A água e o solo são recursos naturais que vêm enfrentando, particularmente no século XXI, complexos impactos nos âmbitos da conservação, aproveitamento e mitigação. Araújo (2018) ressalta que a escassez hídrica e o esgotamento das terras têm acarretado não somente problemas ambientais, mas vêm alcançando patamares nunca antes vistos de natureza socioeconômica.

Conforme Grimaldi et al. (2013), em seus estudos sobre conservação dos solos e da água na África 0cidental, refletem que os valores elevados de precipitações são atualmente a principal causa de erosão e degradação dos solos.

Neste sentido, uma das soluções que visa atender a esfera conservaçãoaproveitamento-mitigação está na alocação de barragens de contenção e infiltração. Esses tipos de estruturas se tornaram ao longo do tempo alternativas econômicas e altamente viáveis, tendo em vista os diferentes tipos de solos, declives e espaços territoriais disponíveis (MIOT0 et al., 2014).

As barraginhas são sistemas dispostos nos solos cujos objetivos são conter as águas das chuvas e fazê-las infiltrarem, assim reduzindo processos degradativos como as erosões, bem como aumentando a disponibilidade hídrica no interior dos solos. Como prática conservacionista, a alocação dessas barragens é considerada vantajosa e de caráter socioambiental (ARAGÃo, 2018b). 
Segundo Pacheco (2016), para a alocação de barraginhas é preciso, previamente, realizar uma identificação de áreas aptas, que ocorre por meio de Sistemas de Informações Geográficas aliados a análises multicritério, para posterior dimensionamento, e, por último, serem então alocadas. No momento que uma dessas etapas é suprimida, os sistemas podem não alcançar as suas máximas eficiências.

Após a etapa de alocação, a etapa de monitoramento e manutenção precisa ser cumprida como determinada. Nota-se com frequência, barraginhas sendo abandonadas por anos, e não há barragem dessa natureza que não necessite de monitoramento e manutenção ao longo dos anos (VERSIANI JÚNIOR, NUZZI E FRÓES, 2007).

No monitoramento, leva-se em consideração a avaliação de três fatores necessários, suas antigas e novas dimensões, análises granulométricas para estabelecimento das texturas e as velocidades de infiltração básica, sendo que as iniciais e finais devem considerar um período de tempo de observação e atuação (FONSECA E SILVA, 2017).

Mas, de modo absoluto, é por meio das velocidades de infiltração básica que 0 pesquisador passa a ter o real panorama do atual funcionamento das barragens de contenção e infiltração. Mesmo com as interdependências de texturas de solo e dimensões, a variável de infiltração é a grande responsável por ratificar ou apontar necessidades de mudanças no dimensionamento original (ARAGÃo, 2018a).

A construção de barraginhas e suas devidas manutenções têm se mostrado etapas indispensáveis quando a abordagem se expressa no âmbito rural, e desde os anos 2000 no Brasil têm levado comunidades e propriedades a ganhos imensuráveis, tanto de ordem financeira e social, quanto de natureza ambiental (VERSIANI JÚNIOR, NUZZI E FRÓES, 2007). Mas, de acordo com Valgma, Torn e Erg (2006), as estruturas de barraginhas, de uma maneira geral, não recebem quantidades adequadas de manutenções, as quais devem ser sempre estabelecidas em projeto.

Como base nestes contextos, mas com foco principal no monitoramento e manutenção de barragens de contenção e infiltração já construídas, esta pesquisa buscou avaliar 0 funcionamento de diferentes barraginhas em relação às suas atuais condições de dimensões, texturas dos solos e velocidades de infiltração.

\section{MATERIAL E MÉTODOS}

Foram selecionadas oito barraginhas localizadas ao sul do município de Montes Claros, área inserida na comunidade rural de Abóboras e com relevância ambiental para o Norte de 
Minas Gerais, tanto pelo desenvolvimento de agriculturas familiares, como pelos importantes recursos hídricos, como o rio São Lamberto e Jequitaí, afluentes do Rio São Francisco.

Seguindo as recomendações de Aragão (2018a), a seleção das barraginhas ocorreu pela forte zona de habitação e agricultura no entorno das barragens, bem como pelas estruturas com maior acúmulo de sedimentos, de acordo com observação direta.

A área de estudo e a localização das barraginhas são mostradas na figura 1, apresentando clima tropical com estação seca de inverno (Aw), conforme classificação de Köppen, e coordenadas de centróide de latitude de $16^{\circ} 54^{\prime} 38$ " S e longitude de $43^{\circ} 56^{\prime} 07$ " 0 , com altitude média de 648 m (ARAGÃo, 2018b).
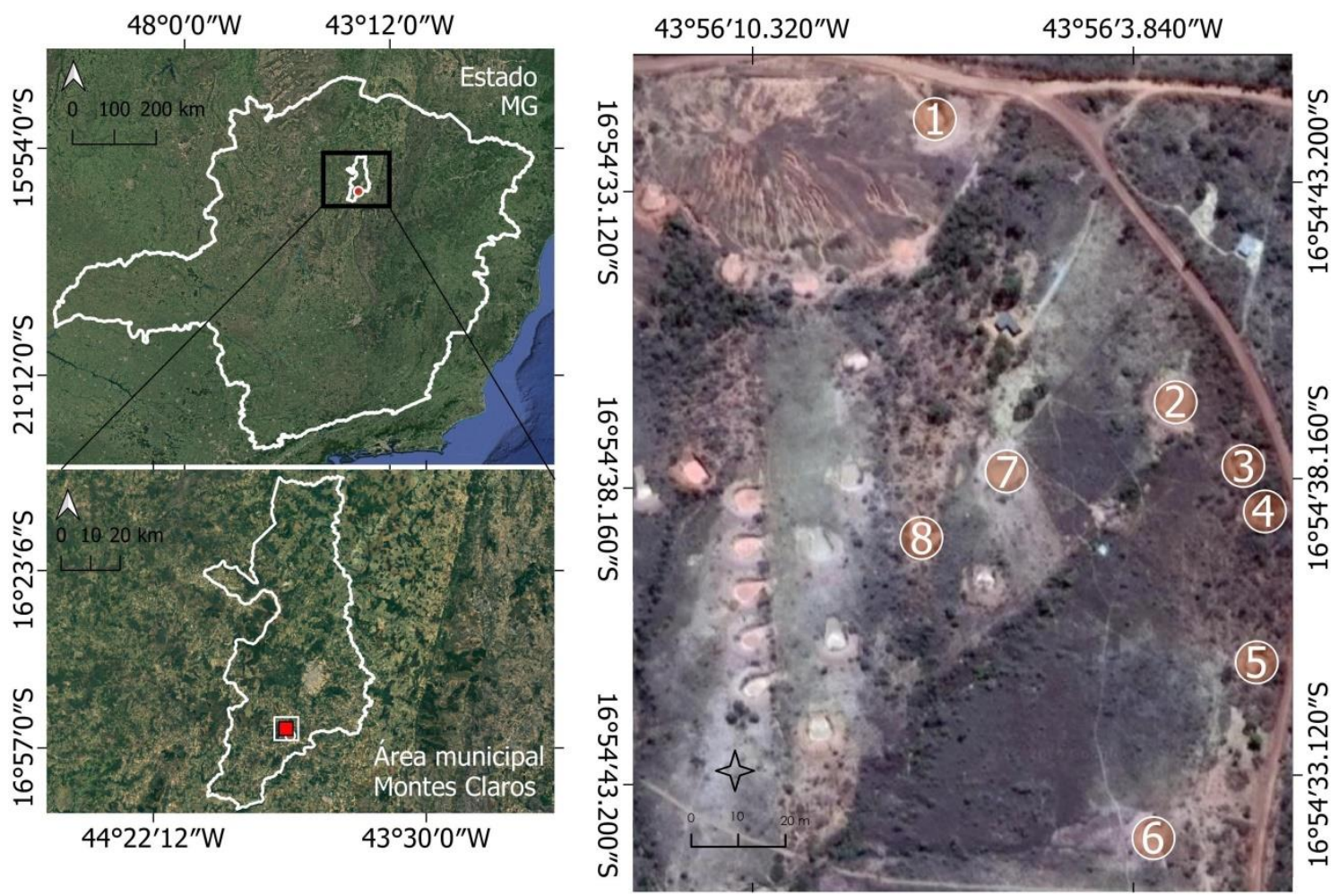

Sistema de Referência de Coordenadas: WGS 84: 4326 | Desenvolvido em 23.05.2019 | Aragão, V. R. | S486672X07374

Figura 1. - Localização das barraginhas.

Fonte: Próprios autores.

As oito barraginhas estão a $25 \mathrm{~km}$ da cidade de Montes Claros, com acesso pela rodovia BR-365, com comunidades limítrofes de Olhos D'Água, Pinheiros e Santa Maria, apresentando uma média pluviométrica de $1.029 \mathrm{~mm}$ e média de temperatura de $22,7^{\circ} \mathrm{C}$, tendo em vista 0 período das últimas três décadas, com ano final sendo o mesmo da realização desta pesquisa, 2018 (VERSIANI JÚNIOR, NUZZI E FRÓES, 2007). 
A vegetação predominante é de gramíneas, com porções esparsas de arbustiva-arbórea do chamado Cerrado sensu lato. A região apresenta latossolos vermelho-amarelos distróficos e cambissolos háplicos distróficos, caracterizando a área como sendo de solos deficientes, relativos à natureza física, química e biológica (ARAGÃ0, 2018b).

A área da pesquisa está inserida na micro-bacia do rio São Lamberto, sub-bacia do rio Jequitaí, na grande bacia do rio São Francisco, região que ganha atenção especial, principalmente pelo município de Montes Claros, desde os anos 2000, com o surgimento das barraginhas no Brasil (VERSIANI JÚNIOR, NUZZI E FRÓES, 2007).

Em campo, realizou-se a coleta dos dados relativos às dimensões das barragens, seguindo o método das extremidades, e as diretrizes do Soil Survey Laboratory Methods, Manual da United States Department of Agriculture (USDA, 2014), aferindo-se os raios e profundidades com auxílio de trenas, resultando assim em duas variáveis.

Em sequência, com o auxílio do Manual de Métodos de Análise de Solo de Teixeira et al. (2017), utilizaram-se trados holandeses em diferentes níveis de profundidade $(15 \mathrm{~cm}$ e $20 \mathrm{~cm}$ ), onde foram coletadas amostras de solo, homogeneizadas e acondicionadas em sacos plásticos transparentes de $10 \mathrm{~cm}$ por $15 \mathrm{~cm}$, devidamente lacrados e transportados para análise laboratorial no Instituto de Ciências Agrárias da Universidade Federal de Minas Gerais, seguindo as diretrizes do Soil Survey Laboratory Methods Manual da United States Department of Agriculture (USDA, 2014).

Em relação às disposições gerais de análises granulométricas de solos designadas por Nobrega et. al. (2018), a análise granulométrica foi obtida por meio do processo de peneiramento das amostras homogeneizadas, seguido da etapa de sedimentação dos sólidos em meio líquido, com secagem do material.

Como etapa final em campo, seguiu-se o método de manuseio de anéis concêntricos para determinar a infiltração básica, em triplicata, juntamente com as metodologias relativas aos infiltrômetros de anéis com base nos estudos de Klein (2014) e Bernardo, Soares e Mantovani (2006), conduzidos no mês de outubro de 2018.

Assim, dois anéis concêntricos de formato cilíndrico foram utilizados para a caracterização da infiltração de água no solo, com diâmetros alternados, sendo o maior de 50 cm e o menor de $25 \mathrm{~cm}$ de diâmetro, ambos com $30 \mathrm{~cm}$ de altura.

Então, os anéis foram cravados no solo com o auxílio de uma marreta de $5 \mathrm{~kg}$, recebendo gradativamente umidificação por um tambor de $20 \mathrm{~L}$, para posterior leitura com régua graduada, num período total de observação de 6 horas fracionadas (8:25 a 14:25), como determinam Aragão (2018a) e Teixeira et al. (2017). 
A carga hidráulica então foi mantida sob 0 mesmo padrão nos cilindros internos e externos, assim permitindo o cálculo das velocidades de infiltração seguindo a expressão potencial do modelo de cálculo de Kostiakov (equação 1), trabalhando-se com os dados: VIBy (Velocidades de Infiltração Básica iniciais e finais); a (constante dependente do solo) e n (variação dependente do solo, de 0 a 1), constante e variável as quais foram estabelecidas em campo com a leitura e interligação de fatores de infiltração pelo manuseio dos anéis concêntricos.

$$
\text { VIBy }=60 * a * n\left[\frac{-0,01}{60 * a * n *(n-1)}\right]^{\frac{(n-1)}{(n-2)}}
$$

Seguindo também as fundamentações de Fagundes et al. (2012), com o objetivo de se padronizar os resultados desta pesquisa, adotou-se o modelo de classificação de VIB de solos proposto da United States Department of Agriculture (USDA, 2014) e por Bernardo, Soares e Mantovani (2006), mostrado na tabela 1.

Tabela 1 - Modelo de classificação de VIB de solos.

\begin{tabular}{cc}
\hline VIB & VIBf $\left(\mathrm{mm}^{-1}\right)$ \\
\hline Muito alta & $>30$ \\
Alta & 15 a 30 \\
Média & 5 a 14 \\
Baixa & $<5$ \\
Fonte: USDA (2014) e Bernardo, Soares e Mantovani (2006).
\end{tabular}

\section{RESULTADOS E DISCUSSÃO}

As barraginhas na comunidade de Abóboras apresentavam um padrão de profundidades de $1,5 \mathrm{~m}$ e raios de $6 \mathrm{~m}$. Assim, com a aplicação da primeira etapa da metodologia desta pesquisa, os resultados atuais de dimensionamentos foram expostos na tabela 2, para compreensão dos fatos de deposição ou retirada de material de solo, onde a identificação de cada barraginha recebeu a sigla Id. 
Tabela 2 - Organização de dados citada no texto

\begin{tabular}{|c|c|c|c|c|}
\hline Id. & Latitude & Longitude & Raio (m) & Profundidade (m) \\
\hline 1 & $16^{\circ} 54^{\prime} 31.89 " \mathrm{~S}$ & $43^{\circ} 56^{\prime} 7.22^{\prime \prime} 0$ & 4,90 & 0,25 \\
\hline 2 & $16^{\circ} 54^{\prime} 36.70^{\prime \prime S}$ & $43^{\circ} 56^{\prime \prime} 3.12^{\prime \prime} 0$ & 4,87 & 0,48 \\
\hline 3 & $16^{\circ} 54^{\prime} 37.78^{\prime \prime S}$ & $43^{\circ} 56^{\prime} 1.97^{\prime \prime} 0$ & 4,80 & 0,31 \\
\hline 4 & $16^{\circ} 54^{\prime} 38.56^{\prime \prime S}$ & $43^{\circ} 56^{\prime} 1.60^{\prime \prime} 0$ & 4,62 & 0,50 \\
\hline 5 & $16^{\circ} 54^{\prime} 41.12^{\prime \prime S}$ & $43^{\circ} 56^{\prime} 1.74^{\prime \prime} 0$ & 4,89 & 0,18 \\
\hline 6 & $16^{\circ} 54^{\prime} 44.11 " \mathrm{~S}$ & $43^{\circ} 56^{\prime} 3.50^{\prime \prime} 0$ & 4,64 & 0,31 \\
\hline 7 & $16^{\circ} 54^{\prime} 37.88^{\prime \prime S}$ & $43^{\circ} 56^{\prime} 6.00^{\prime \prime} 0$ & 4,33 & 0,25 \\
\hline 8 & $16^{\circ} 54^{\prime} 39.01 " \mathrm{~S}$ & $43^{\circ} 56^{\prime} 7.45^{\prime \prime} 0$ & 4,88 & 0,34 \\
\hline \multicolumn{3}{|c|}{ Média } & 4,74 & 0,32 \\
\hline
\end{tabular}

Pela Tabela 2 é possível observar que os raios das barraginhas sofreram redução, o que pode ser explicado pela sedimentação das partículas de solos em suas bordas superficiais. Desta forma, pode-se afirmar que por mais que as barragens de infiltração tenham em algum momento sofrido processos de intemperismo, o predomínio foi do acúmulo de sedimentos.

Este mesmo padrão de sedimentação também fica evidente em relação à variável de profundidade, uma vez que os valores, que inicialmente em 2007 eram de 1,5 m, sofreram drásticas reduções, o que caracteriza a deposição de materiais de solos no interior das barraginhas. É importante ressaltar que em todo o período de 2007 a 2018, os sistemas não passaram por manutenções.

Tendo em vista uma variação média de raio de $4,74 \mathrm{~m}$ e de profundidade de $0,32 \mathrm{~m}$ no período analisado, seguindo as instruções normativas de Teixeira et al. (2017), sobre manutenção de barraginhas por meio do raio e profundidade, recomenda-se a cada cinco anos que sejam realizadas escavações para que a profundidade seja adequada ao mínimo de 1,5 m e máximo de 2,0 m, para que se possa obter a máxima eficiência do sistema, também como argumentam Fagundes et al. (2012) e o memorial de dimensionamento de Aragão (2018a).

Em relação aos solos, os resultados das análises granulométricas realizadas no Instituto de Ciências Agrárias da Universidade Federal de Minas Gerais permitiram identificar a tendência de cada tipo de solo em função de suas texturas, conforme mostrado na tabela 3. 
Tabela 3 - Solos e texturas das barraginhas

\begin{tabular}{llllll}
\hline Id. & Areia grossa $\left(\% \cdot \mathbf{k}^{-\mathbf{l}}\right)$ & Areia fina $\left(\% \cdot \mathbf{k}^{-\mathbf{1}}\right)$ & Silte $\left(\% \cdot \mathbf{k}^{-\mathbf{1}}\right)$ & Argila $\left(\% \cdot \mathbf{k}^{-\mathbf{1}}\right)$ & Textura \\
\hline 1 & 2,90 & 81,10 & 8,00 & 8,00 & Arenosa \\
2 & 5,90 & 48,10 & 36,00 & 10,00 & Arenosa \\
3 & 2,00 & 54,00 & 30,00 & 14,00 & Média \\
4 & 0,80 & 57,20 & 30,00 & 12,00 & Arenosa \\
5 & 0,10 & 71,90 & 16,00 & 12,00 & Arenosa \\
6 & 0,01 & 64,00 & 24,00 & 12,00 & Arenosa \\
7 & 3,30 & 54,70 & 30,00 & 12,00 & Arenosa \\
8 & 0,10 & 37,90 & 44,00 & 18,00 & Média \\
\hline
\end{tabular}

Fonte: Próprios autores.

Leal e Tonello (2016) argumentam que o solo é um elemento essencial ao designar uma área suscetível a problemas ambientais, principalmente em um sistema de captação de água de chuva, onde parâmetros como a estrutura da superfície, a taxa de infiltração e a percolação, a profundidade do solo e sua textura devem ser levados em consideração. Infiltrar é, sem dúvidas, o objetivo fundamental e prioritário das barraginhas.

Tendo em vista essa consideração, as barraginhas de número 3 e 8 apresentaram uma tendência de percolação menor em relação às demais avaliadas nesta pesquisa, apresentando texturas médias, e, portanto, menores espaços de vazios.

Já os solos arenosos, presentes nas barraginhas 1, 2, 4, 5, 6 e 7, foram caracterizados como mais leves e com maior consistência granulosa, compostos por grãos grossos, médios e finos, visíveis a olho nu, com grandes espaços de poros, designados macroporos, proporcionando uma maior drenagem nesses perfis para as águas pluviais.

Ralisch et al. (2017) expõem em seus estudos sobre estrutura de solos, que a tendência arenosa facilita a percolação hídrica, fazendo com que haja, mais rapidamente, uma condição de saturação, e assim, evidencia-se naturalmente o mesmo padrão para situações onde a água passa a secar no interior dos mesmos.

Os resultados quanto à textura mostram que por mais que os solos apresentem uma tendência arenosa, com consequente maior capacidade de desprendimento e carreamento de partículas, a região do estudo, por possuir uma intensa utilização de irrigação por meio de poços artesianos, beneficia-se da rápida infiltração. Desta forma, há uma rápida recarga dos lençóis freáticos, evitando-se os processos de rebaixamento (VLAAR, 1992). 
Em relação às taxas de infiltração básica, técnicas simples podem ser aplicadas, desde que aptas a retratarem adequadamente a situação real do solo, naquele momento. Dentre as técnicas de campo, a mais utilizada é a de anéis concêntricos, devido à simplicidade e praticidade (ARAGÃO, 2018a).

Os resultados obtidos nesta pesquisa sobre as VIBy foram representados na tabela 4 demonstrando que, após certo ponto, a tendência de percolação da água nos solos decai e tende ao equilíbrio.

Tabela 4 - VIBi e VIBf das barraginhas.

\begin{tabular}{lllllllll}
\hline VIBi & $\mathbf{1}$ & $\mathbf{2}$ & $\mathbf{3}$ & $\mathbf{4}$ & $\mathbf{5}$ & $\mathbf{6}$ & $\mathbf{7}$ & $\mathbf{8}$ \\
$08: 25$ & 180,33 & 177,66 & 96,33 & 177,99 & 178,66 & 179,22 & 181,66 & 96,99 \\
\hline VIBf & $\mathbf{1}$ & $\mathbf{2}$ & $\mathbf{3}$ & $\mathbf{4}$ & $\mathbf{5}$ & $\mathbf{6}$ & $\mathbf{7}$ & $\mathbf{8}$ \\
$14: 25$ & 22,33 & 22,66 & 11,33 & 21,99 & 22,66 & 23,22 & 24,66 & 11,99 \\
\hline
\end{tabular}

Fonte: Próprios autores.

Diante da tabela 4 fica evidente que as informações relativas à VIBy se intercomunicam com as análises granulométricas dos solos, ratificando que os solos das barraginhas escolhidas para o estudo tendem a favorecer a percolação, objetivo fundamental e prioritário de bacias de contenção e infiltração das águas das chuvas. Contudo, os sedimentos revelados pelas medições de profundidade juntamente com as leituras de VIBy mostram uma sobrecarga dos sistemas.

As características de tendência à percolação facilitada dos solos da região podem ser explicadas também tendo em vista o estudo de Aragão (2018b), no qual estabeleceu que no local predominam solos distróficos, com deficiências em suas ordens físicas e químicas, com destaque para o Cambissolo Háplico Tb Distrófico e o Latossolo Vermelho-Amarelo Distrófico.

Com base na classificação de Bernardo, Soares e Mantovani (2006), os solos analisados neste estudo se apresentaram nas barraginhas 3 e 8 como de VIB média, por estarem enquadrados em VIBf entre 5 e $14 \mathrm{~mm} \cdot \mathrm{h}^{-1}$, resultados estes que confirmam uma sobrecarga operante nas estruturas.

Aragão (2018a) argumenta que, para sistemas que dependem de infiltração das águas nos solos, quanto mais próxima de $30 \mathrm{~mm} . \mathrm{h}^{-1}$ é a VIBf, melhor o sistema irá funcionar, como é 0 caso das barraginhas. Neste contexto, Viana, Clemente e Oliveira (2016) ressaltam que esta condição essencialmente ideal é um tanto quanto complicada de ocorrer. 
Convergindo às fundamentações dos autores supracitados, as barragens de contenção e infiltração número 1, 2, 4, 5, 6 e 7 foram as que mais se aproximaram do valor considerado ideal (30 mm.h $\mathrm{h}^{-1}$. Contudo, ainda com notável comprometimento de infiltração, a que apresentou maior taxa de VIBf, 24,66 mm.h-1 foi a barraginha 7 .

Assim, conforme a classificação de Bernardo, Soares e Mantovani (2006), as VIB das barraginhas 1, 2, 4, 5, 6 e 7 foram enquadradas com velocidades de infiltração básicas consideradas altas, ou seja, entre 15 e $30 \mathrm{~mm} \cdot \mathrm{h}^{-1}$.

\section{CONCLUSÃ0}

Os resultados demonstram que na região da comunidade de Abóboras, as barraginhas analisadas estão operando com sobrecarga. Suas texturas estão dentro dos padrões estabelecidos na literatura científica, a qual foi abordada nesta pesquisa. As barraginhas apresentam uma forte ligação entre as dimensões atuais e os níveis de infiltração básica.

Desta forma, as barragens de contenção e infiltração demonstraram a necessidade de manutenções, mesmo não tendo apresentado eventos atípicos e extremos de natureza degradativa, e considerando que os solos da região possuem distrofias, com predomínio do Cambissolo Háplico Tb distrófico e do Latossolo Vermelho-Amarelo distrófico.

Através dos estudos científicos adotados como referência nesta pesquisa, mesmo sem acesso completo ao projeto de dimensionamento, foi possível sugerir uma periodicidade estimada de cinco anos para manutenções nas barraginhas avaliadas. Assim, reafirma-se a importância desses sistemas cumprirem corretamente os prazos de monitoramento e manutenções estabelecidos em projeto.

Pelo fato das barraginhas terem em seu interior solos expostos às condições de tempo e clima, é evidente que um mesmo estudo, considerando naturezas diferentes de uso e ocupação, fornecerá resultados que não podem ser comparados, principalmente com relação às taxas de infiltração básica. Esta pesquisa não é, portanto, um modelo metodológico fechado, mas uma base para possíveis novos estudos, a fim de se buscar saudáveis contraposições e novos caminhos.

\section{REFERÊNCIAS BIBLIOGRÁFICAS}

ARAGÃo, V. R. Sanding of nitrates: memorial of calculations. Municipal Secretary of the Environment of Montes Claros, 2018a. 1 CD-ROM. 
ARAGÃo, V. R. Identificação de áreas aptas a barraginhas na bacia do rio São Lamberto. 2018. 20 f. TCC (Graduação) - Curso de Graduação em Engenharia Ambiental e Sanitária, Instituto Educacional Santo Agostinho, Montes Claros, 2018b.

ARAÚJO, T. M. Caracterização hidroambiental de uma microbacia hidrográfica da bacia do rio São Mateus, no Vale do Mucuri, Minas Gerais. 2018. 105 f. Dissertação (Mestrado) - Curso de Pós-graduação em Ciência Florestal, Universidade Federal de Viçosa, Viçosa, 2018.

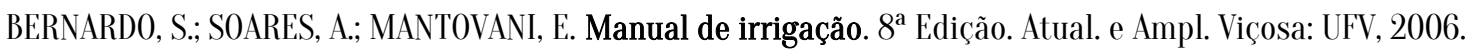

FAGUNDES, E. A. A.; KOETZ, M.; RUDEL, N.; SANTOS, T. S.; PORTO, R. Determinação da infiltração e velocidade de infiltração de água pelo método de infiltrômetro de anel em solo de cerrado no município de Rondonópolis-MT. Enciclopédia Biosfera, Centro Científico Conhecer - Goiânia, v.8, N.14; p. 369-378, 2012.

FONSECA, E. L.; SILVA, E. P. Análise fisiográfica como subsídio ao estudo da suscetibilidade erosiva em bacias hidrográficas. Acta Geográfica, v. 11, n. 25, p. 137-158, 2017.

GRIMALDI, S.; ANGELUCCETTI, I.; COVIELLO, V.; VEZZA, P. Cost-Effectiveness of Soil and Water Conservation Measures on the Catchment Sediment Budget-The Laaba Watershed Case Study. Land Degradation \& Development, n. 26, v. 7, p. 737-747, 2013.

KLEIN, V. A. Física do solo. $3^{\text {a }}$ ed. Passo Fundo: Ed. da EDIUPF, 2014.

LEAL, M. S.; TONELLO, K. C. Análise da morfometria e do uso e cobertura da terra da microbacia do córrego Ipaneminha de Baixo. Floresta, v. 46, n. 4, p. 439-446, 2016.

MIOT0, C. L. et al. Morfometria de bacias hidrográficas através de SIGs livres e gratuitos. Anuário do Instituto de Geociências, v. 37, n. 2, p. 16-22, 2014.

NOBREGA, G. N.; ROMER0, D. J.; OTER0, X. L.; FERREIRA, T. O. Pedological Studies of Subaqueous Soils as a Contribution to the Protection of Seagrass Meadows in Brazil. Rev. Bras. Ciênc. Solo, vol. 42, p. 1-12, 2018.

PACHEC(, F. F. Análise Multicritério aplicada na seleção de áreas aptas a construção de barraginhas em relevo forte ondulado. 2016. 33 f. TCC (Graduação) - Curso de Graduação em Geografia, Departamento de Geografia, Universidade Federal de Viçosa, Viçosa, 2016.

RALISCH, R.; DEBIASI, H.; FRANCHINI, J. C.; TOMAZI, M.; HERNANI, L. C.; MELO, A. da S.; SANTI, A.; MARTINS, A. L. da S.; BONA, F. D. de. Diagnóstico Rápido da Estrutura do Solo - DRES. Londrina: Embrapa Soja, 2017.

TEIXEIRA, P. C.; DONAGEMMA, G. K.; FONTANA, A.; TEIXEIRA, W. G. Manual de métodos de análise de solo. $3^{a}$ ed. Brasília: Embrapa Solos, 2017.

USDA - United States Department of Agriculture. Soil Survey Laboratory Methods Manual. Lincoln: Natural Resources Conservation Service Soils, 2014.

VALGMA, I.; TORN, H.; ERG K. The impact of infiltration dam on the groundwater regime in the Kurtna landscape reserve area. 0il Shale Estonian Academy Publishers, n.l, vol. 23, p. 3-14, 2006.

VERSIANI JÚNIOR, E. R.; NUZZI, G. A. R.; FRÓES, A. R. Projeto de Revitalização da Microbacia do Rio Abóboras Bacia Hidrográfica São Lamberto. In: $6^{\circ}$ Simpósio Brasileiro de Captação e Manejo de Água de Chuva, 2007, Belo Horizonte. Anais... Belo Horizonte: ABCMAC, 2007.

VLAAR, J. C. J. Design and effectiveness of permeable infiltration dams in Burkina Faso. Land Degradation $\mathcal{E}$ Development, n.1, vol. 3, p. 37-53, 1992.

VIANA, J. H. M.; CLEMENTE, E. de P.; OLIVEIRA, A. P. de. Procedimento operacional padronizado para quantificação e mensuração de areias via análise de imagens. Rio de Janeiro: Embrapa Solos, 2016. 
